\title{
The changing face of rheumatic fever in the 20th century
}

\begin{abstract}
Streptococcus pyogenes is a natural pathogen only for human beings. Its surface is composed of tiny hairy projections by which the organism adheres to human tissues. In virulent strains, these structures are heavily coated by the remarkably heterogeneous and immunologically specific $M$ protein [1], of which $>90$ serotypes have so far been identified. When fully virulent, each chain is surrounded by a capsule of hyaluronic acid responsible for the appearance of 'mucoid' colonies on blood agar. Purified streptococcal hyaluronate is chemically identical to that found in the human host and antibodies to it are difficult to raise.
\end{abstract}

$\mathrm{M}$ protein and capsule are both responsible for the striking resistance to phagocytosis of virulent strains. However, it is the anti-M immune response that confers solid and long-lasting immunity, but only to a single serotype. Both virulence factors are rapidly lost during convalescence from acute pharyngitis, or when grown in artificial media. However, strain virulence may be maintained by frequent intraperitoneal mouse passage or frequent transfer through fresh human blood. On blood agar, capsulate variants form large mucoid colonies resembling a drop of oil, whereas loss of the capsule results in colonies that are opaque and 'pearly' in appearance. Considering the potential significance of this observation, it is surprising how rarely clinical microbiology laboratories recognise and report the presence of mucoid colonies on throat cultures. Their appearance in military cohorts under prospective surveillance often presages severe epidemics of streptococcal pharyngitis and rheumatic fever (RF) [2,3]. Strains lacking both virulence properties are no more harmful than commensals, and once strains have dissociated, virulence is not recoverable in vitro.

Pharyngeal carriage may persist stubbornly during convalescence, and these strains, devoid of M protein and capsules, may retain major adherence ligands, such as lipoteichoic acid. Thus, the human throat is readily colonised by strains of low or no virulence, often causing false positive throat cultures in patients with sore throats due to viruses. Confirmation of the diagnosis of streptococcal pharyngitis therefore requires evidence of a significant rise in streptococcal antibody titres (e.g., antistreptolysin O). In practice, it is the predictive power of a negative throat culture to exclude group A streptococcal infection that best supports a decision to forego antibiotic therapy in acute pharyngitis [4].

The World War II epidemics of RF that occurred among military recruits assembled from many parts of the USA were caused by strains of $S$. pyogenes belonging to several prevalent $M$ serotypes. These epidemic strains were capsulate, M-protein-rich variants that spread rapidly among close-living recruits. Infections with several $\mathrm{M}$ serotypes were associated with a strikingly similar RF attack rate of c. $3 \%$ in the untreated individuals who participated in the classic controlled studies of the penicillin regimens that prevented RF [5].

Subsequently it became clear that streptococcal sore throats in some civilian settings were associated with little or no RF. Such low frequencies occurred despite the usual high prevalence of streptococcal pharyngitis in school children and failure to use the rigorous penicillin regimens required for effective RF prevention. It was suggested that rheumatogenic strains had been driven out of military bases and civilian communities by improved diagnosis, enthusiastic (and undiscriminating) antibiotic treatment of all upper respiratory infections and improved living conditions $[2,6]$. Only a limited number of $M$ serotypes had been associated with epidemic RF, and mucoid group A streptococci belonging to these $\mathrm{M}$ types seemed to have disappeared $[3,7]$.

The argument for qualitative differences between rheumatogenic and non-rheumatogenic strains was supported by studies of epidemics of impetigo caused by so-called 'skin strains' $[8,9]$. These belonged to $M$ and $\mathrm{T}$ serotypes other than those associated with $\mathrm{RF}$ and infection or carriage did not result in RF. In communities in the southern USA, RF was very rare in summer despite prevalent impetigo [10]. Moreover, some impetigo strains also caused acute glomerulonephritis (AGN). In fact, rheumatogenic throat strains do not cause concurrent epidemics of RF and AGN and these post-streptococcal complications are hardly ever observed in the same individual at the same time. Although AGN may be caused by either skin or throat strains, RF occurs only after pharyngeal infection by certain virulent strains. However, strain variation 
occurs within a given $M$ serotype [11], so that $M$ serotype alone does not necessarily imply rheumatogenicity.

In the 1970s, the virtual disappearance of acute and recurrent RF in the developed countries of the world made it difficult to identify rheumatogenic strains $[12,13]$. There was no significant decrease in the prevalence of endemic streptococcal sore throat in the school children of these countries [14], but the M serotypes most commonly associated with RF virtually disappeared [15]. In the 1980s there was a resurgence of RF in some military and civilian communities of the USA, associated with mucoid strains belonging to the notorious $M$ serotypes of former RF epidemics. They appeared to be well disseminated among cohorts of RF subjects [16].

By the 1980s, the primary molecular structure of $\mathrm{M}$ protein was determined and its type-specific antigen shown to reside in its small terminal $\mathrm{N}$-acetyl peptide [17]. Two highly conserved epitopes within $M$ protein divided group A streptococci immunologically into class I (throat) and class II (skin) strains [18]. All RF strains fell clearly into class I throat strains and individuals who had contracted RF had higher than normal titres of antibodies to the class I epitope, but lacked antibodies to the class II epitope [19]. Moreover, in the large $M$ protein molecules of class I rheumatogenic strains, distinct epitopes were identified that cross-reacted with cardiac, synovial and brain tissues and these were separable from the terminal type-specific antigen [20].

The genes of $\mathrm{M}$ protein $(\mathrm{emm})$ are divided into four subfamilies, arranged in five distinctive chromosomal patterns, designated A-E [21]. These genetic patterns clearly differentiate rheumatogenic pharyngitis strains from impetigo strains, but somewhat less clearly from other throat strains. Whether epitopes that cross-react with host tissues are present exclusively in RF strains is not yet clear. An attractive hypothesis is that the deposition of a heavy antigenic load of these crossreactive epitopes in pharyngeal lymphoid tissues, already hypersensitised during early childhood by repeated streptococcal infections, causes a break in the immune tolerance of susceptible hosts that leads to the various stigmata of RF.

Swallowing large amounts of such antigens seems to have a powerful immunising effect. It is an old observation that streptococcal pharyngitis produces an immune response to all streptococcal antigens that is more striking in those who develop RF than in those wbo do not; in contrast, RF patients respond normally to other antigens to which they have been tested. After nasal administration of synthetic $M$ vaccines, mice produce type-specific IgA antibodies and are protected from systemic challenge with homologous M-type strains [22]. Furthermore, the adjuvant effect of the superantigenic properties of the proximal part of the $\mathrm{M}$ protein (see below) may also promote exaggerated immune responses and autoimmunity.

By DNA fingerprinting of group A streptococci, and by DNA sequencing of the emm genes of $\mathrm{M}$ proteins, strains belonging to the same $M$ serotype may show distinct variations despite their close genetic similarity [23]. So far, rheumatogenic strains are found among a limited number of $\mathrm{M}$ serotypes, most notoriously within M5 [7]. Anti-M immunity focused on these serotypes might prevent much RF [20].

Strains of S. pyogenes associated with toxic shock and necrotising fasciitis reappeared in the USA in the mid1980s and have spread throughout the world [23]. At the same time, RF began to decline again, so that the prevalence of the two diseases does not appear to be epidemiologically congruent [24]. Over half the toxic strains isolated from invasive infections, belong to $\mathrm{Ml}$ or M3 serotypes. They often produce one of the scarlet fever-associated streptococcal pyrogenic exotoxins, SPE A, which has close molecular homology with the exotoxin of staphylococcal toxic shock, enterotoxin B [25]. These pyrogenic exotoxins, like proximal parts of the $\mathrm{M}$ protein molecule, are superantigens, compounds that activate T-lymphocyte receptors non-specifically and act as powerful immunological adjuvants $[26,27]$.

The clonal nature of invasive streptococcal infections is further illustrated by a recent survey of Ml strains from 13 countries on five continents. Although these strains showed substantial genetic diversity, most invasive infections were caused by two subclones [24]. One such strain which has been isolated worldwide has the speA gene. However, in a recent report from Japan, although $\mathrm{Ml}$ and M3 serotypes accounted for more than half of the invasive strains studied, they did not contain the SPE A toxin [28]. Apparently other exotoxins remain to be identified, if these are indeed responsible for the toxic shock syndrome. In Minneapolis, Minnesota, an outbreak of invasive $S$. pyogenes infections was due to the spread of a single clone identified as such by sequencing the genes of its $\mathrm{M}$ protein $(\mathrm{emm})$ and its pyrogenic toxin [29].

Although the specific pathogenic substance(s) and host factors causing RF are still unknown, the importance of specific anti-M immunity is clear. The type-specific epitope contained in the $\mathrm{N}$-acetyl amino terminus of purified $\mathrm{M}$ protein can now be completely separated from the molecular moieties cross-reacting with host tissues, making possible the development of vaccines free of such possibly dangerous antigens. Recently, Dale and his colleagues [30] have constructed recombinant multivalent $M$ protein vaccines that evoke, in rabbits, bactericidal antibodies against all the serotypes represented. Most important, these antibodies do not cross-react with human tissues. 
Such a vaccine for human use is under trial (J. Dale, personal communication). In the absence of an animal reservoir, effective multivalent $\mathrm{M}$ immunisation focused on areas where RF is epidemic or heavily endemic might ultimately eradicate the disease from the world. If and when new rheumatogenic $M$ types appear, microbiologists should be ready to identify them for inclusion in a vaccine, as they have done for multivalent vaccines designed for other diseases. And, of course, if the putative host factor(s) of RF are ever revealed, the target for an RF vaccine could be sharply focused.

Meanwhile, the best defence against $R F$ is a heightened awareness that the identification of a single case of RF or streptococcal toxic shock is a signal that a dangerous clone of $S$. pyogenes has appeared; it should be greeted like a plague and treated accordingly!

G. H. STOLLERMAN

Professor of Medicine and Public Health Emeritus, Boston University,

10 Willow Springs Lane, Apartment 21, Hanover, NH 03755, USA

E-mail: gstollerman@compuserve.com

\section{References}

1. Lancefield RC. Specific relationship of cell composition to biological activity of hemolytic streptococci. Harvey Lect 1941; 35: $251-290$.

2. Stollerman GH. Rheumatic fever. Lancet 1997; 349: 935-942.

3. Stollerman GH. Changing streptococci and prospects for the global eradication of rheumatic fever. Perspect Biol Med 1997; 40: $165-189$

4. Bisno AL, Gerber MA, Gwaltney JM, Kaplan EL, Schwartz RH. Diagnosis and management of group A streptococcal pharyngitis: a practice guideline. Clin Infect Dis 1997; 25: $574-583$.

5. Rammelkamp CH, Denny FW, Wannamaker LW. Studies on the epidemiology of rheumatic fever in the armed services. In: Thomas L (ed) Rheumatic fever. Minneapolis: University of Minnesota Press. 1952: 72-89.

6. Stollerman GH, Siegel AC, Johnson EE. Variable epidemiology of streptococcal disease and the changing patterns of rheumatic fever. Mod Concepts Cardiovasc Dis 1965; 34: 45-48.

7. Bisno AL. The concept of rheumatogenic and non-rheumatogenic group A streptococci. In: Read SE, Zabriskie JB (eds) Streptococcal diseases and the immune response. New York, Academic Press. 1980: 789-803.

8. Top FH, Wannamaker LW, Maxted WR et al. M antigens among group A streptococci isolated from skin lesions. $J$ Exp Med 1967; 126: 667-685.

9. Wannamaker LW. Differences between streptococcal infections of the throat and of the skin. (In two parts). $N$ Engl $J$ Med 1970; 282: 23-31, 78-85.

10. Bisno AL, Pearce IA, Wall HP, Moody MD, Stollerman GH Contrasting epidemiology of acute rheumatic fever and acute glomerulonephritis. Nature of the antecedent streptococcal infection. $N$ Engl J Med 1970; 283: 561-565.

11. Maxted WR, Valkenburg HA. Variation in the M-antigen of group-A streptococci. J Med Microbiol 1969; 2: 199-210.

12. Land M, Bisno AL. Acute rheumatic fever: a vanishing disease in suburbia. JAMA 1983; 249: 895-898.

13. Bisno AL, Pearce IA, Stollerman GH. Streptococcal infections that fail to cause recurrences of rheumatic fever. $J$ Infect Dis 1977; 136: 278-285.

14. Holmberg SD, Faich FA. Streptococcal pharyngitis and acute rheumatic fever in Rhode Island. JAMA 1983; 250: 2307-2312.

15. Schwartz B, Facklam RR, Breiman RE. Changing epidemiology of group A streptococcal infection in the USA. Lancet 1990; 336: $1167-1171$.

16. Centers for Disease Control. Acute rheumatic fever Utah. MMWR 1987; 36: 108.

17. Beachey EH, Stollerman GH, Johnson RH, Ofek I, Bisno AL Human immune response to immunization with a structurally defined polypeptide fragment of streptococcal M protein. J Exp Med 1979; 150: 862-877.

18. Bessen DE, Jones KE, Fischetti VA. Evidence for two distinct classes of streptococcal M-protein and their relationship to rheumatic fever. J Exp Med 1989; 169: 269-283.

19. Bessen DE, Veasy LG, Hill HR, Augustine NH, Fischetti VA. Serologic evidence for a class I group A streptococcal infection among rheumatic fever patients. $J$ Infect Dis 1995; 172: $1608-1611$.

20. Dale JB, Simmons M, Chiang BC, Chiang BY. Recombinant octavalent group A streptococcal vaccine. Vaccine 1996; 14: 944-948.

21. Bessen DE, Sotir CM, Readdy TL, Hollingshead SK. Genetic correlates of throat and skin isolates of group A streptococci. $J$ Infect Dis 1996; 173: 896-900.

22. Dale JB, Chiang BC. Intranasal immunization with recombinant group A streptococcal M fragment fused to the B subunit of Escherichia coli labile toxin protects mice against systemic challenge infections. $J$ Infect Dis 1995; 171: 1038-1041.

23. Musser JM, Kapur V, Szeto J, Pan X, Swanson DS, Martin DR. Genetic diversity and relationships among Streptococcus pyogenes strains expressing serotype Ml protein: recent intercontinental spread of a subclone causing episodes of invasive disease. Infect Immun 1995; 63: 994-1003.

24. Kaplan EL. Global assessment of rheumatic fever and rheumatic heart disease at the close of the century. Circulation 1993; 88: $1964-1972$.

25. Nelson K, Schlievert PM, Selander RK, Musser JM. Characterization and clonal distribution of four alleles of the spe $A$ gene encoding pyrogenic exotoxin A (scarlet fever toxin) in Streptococcus pyogenes. J Exp Med 1991; 154: 1271-1274.

26. Watanabe-Ohnishi R, Aelion J, LeGros L et al. Characterization of unique human TCR V specificities for a family of streptococcal superantigens represented by rheumatogenic serotypes of M protein. J Immunol 1994; 152: 2066-2073.

27. Mollick JA, Miller GG, Musser JM, Cook RG, Grossman D, Rich RR. A novel superantigen isolated from pathogenic strain of Streptococcus pyogenes with aminoterminal homology to staphylococcal enterotoxin B and C. J Clin Invest 1993; 92 $710-719$.

28. Nakashima $\mathrm{K}$, Ichiyama $\mathrm{S}$, Imuma $\mathrm{Y}$ et al. A clinical and bacteriologic investigation of invasive streptococcal infections in Japan on the basis of serotypes, toxin production, and genomic DNA fingerprints. Clin Infect Dis 1997; 25: 260-266.

29. Cockerill FR, MacDonald KL, Thompson RL et al. An outbreak of invasive group A streptococcal disease associated with high carriage rates of the invasive clone among schoolaged children. JAMA 1997; 277: 38-43.

30. Dale JB. Multivalent group A streptococcal vaccine designed to optimize the immunogenicity of six tandem $M$ protein fragments. Vaccine 1998 (in press) 